\title{
Virus-like particles of Macrobrachium rosenbergii nodavirus produced in bacteria
}

\begin{abstract}
Macrobrachium rosenbergii nodavirus (MrNv) infects giant freshwater prawns and causes white tail disease (WTD). The coding region of the capsid protein of $\mathrm{MrNv}$ was amplified with RT-PCR and cloned into the pTrcHis2-TOPO vector. The recombinant plasmid was introduced into Escherichia coli and protein expression was induced with IPTG. SDS-PAGE showed that the recombinant protein containing the His-tag and myc epitope has a molecular mass of about $46 \mathrm{kDa}$ and it was detected by the anti-His antibody in Western blotting. The protein was purified using immobilized metal affinity chromatography (IMAC) and transmission electron microscopic analysis revealed that the recombinant protein assembled into VLPs.
\end{abstract}

Keyword: Virus-like particles; Nodavirus; Nodaviridae; Cloning; Purification; Expression 